\title{
Surface Electromyographic Study of Peroneus Longus Activation during Ankle Eversion and Heel Lift
}

\author{
Jian Chen, Robert Jack, Jessica Appelle, Michael Bushuk, Camille Smith \\ School of Human Kinetics, Laurentian University, Sudbury, Ontario, Canada \\ Email: jchen@laurentian.ca
}

Received 19 June 2015; accepted 8 August 2015; published 11 August 2015

Copyright (C) 2015 by authors and Scientific Research Publishing Inc.

This work is licensed under the Creative Commons Attribution International License (CC BY).

http://creativecommons.org/licenses/by/4.0/

cc) (†) Open Access

\section{Abstract}

Prophylactic and rehabilitative ankle training programs rarely incorporate ankle eversion exercises of the weight bearing leg during one-legged stand. Such training may be necessary for the optimal PL function and dynamic ankle control. In this study, we compared a PL exercise that involved eversion of the inverted ankle of a weight bearing leg against the heel lift (HL), a popular PL strengthening exercise. The PL activation patterns were studied in 20 university students (7 male and 13 female). The self-reported dominant legs of the subjects were tested in this study. After recording the surface electromyographic (EMG) activity level of their PL maximal voluntary isometric contractions (MVIC), the subjects performed HL, and ankle eversions of the weight bearing ankle during one-legged stand. Ankle eversions were initiated from two inverted ankle positions, 20 degree inversion (EV1) and 25 degree inversion (EV2). The ankle position and the PL muscle activity were recorded with an accelerometer affixed to the dorsum of the foot and a surface EMG electrode over PL respectively. The ranges of motion for HL, EV1, and EV2 were 25, 29, and 31 degrees respectively. Expressed as percentage of MVIC, the average normalized EMG linear envelopes for EV1 $(73 \%)$ and EV2 $(74 \%)$ were significantly greater than HL $(64 \%)$. These results demonstrated that eversions of the inverted ankle of the weight bearing leg elicited stronger PL activation that may result in better lateral ankle strength and dynamic control. Incorporation of weight bearing ankle eversions may enhance effectiveness of PL exercise and balance.

\section{Keywords}

Strengthening, Ankle Injury, EMG, Rehabilitation

\section{Introduction}

In the event of a sudden ankle inversion, peroneus longus (PL) plays a key role in preventing or limiting the se-

How to cite this paper: Chen, J., Jack, R., Appelle, J., Bushuk, M. and Smith, C. (2015) Surface Electromyographic Study of Peroneus Longus Activation during Ankle Eversion and Heel Lift. Open Journal of Therapy and Rehabilitation, 3, $101-108$. 
verity of a lateral ankle sprain [1]. Biomechanical study indicates the prime importance of the PL strength in limiting ankle inversion as compared with mechanical barrier effect provided by various functional ankle orthoses and taping [2]. Optimal function of this evertor muscle depends on its strength [3], timely activation [4] [5], proprioception [6], and central modulation of motor control [7].

An effective functional exercise for PL may require several contextual elements that include the eversion of an inverted ankle, weight bearing, and postural control. These elements approximate the circumstance when PL is called to task and have been shown to evoke strong peroneal activation [8] [9]. Furthermore, deficits associated with injured or unstable ankles may be more effectively addressed through ankle eversion exercises that incorporate postural control and weight bearing. There are evidences that ankle instability may be associated with functional deficits of peroneal muscles under weight-bearing condition. Individuals with a history of ankle injury and functional ankle instability exhibited diminished peroneal activity in the weight bearing leg condition [10] as well as a delayed and decreased motor response to ankle inversion [4] [5]. These deficits may be partly related to poor postural control in persons with ankle injury. Consistent with this view is the phenomenon of reciprocal influence between the ankle injury and the impaired postural control. Individuals with an acute ankle sprain or with a history of repetitive ankle sprains suffered from impaired postural control during single-leg standing [11]-[14]. On the other hand, poor balance performance was highly predictive of future injury risk in prospective studies [15]-[17]. Therefore, in order to mitigate ankle injury related deficits and to reduce the risk of ankle injury, an argument can be made for peroneal strengthening exercises to be carried out in the context of a weight-bearing inverted ankle and postural control.

PL exercises that are commonly performed include resistive ankle eversion with cuff and elastic tubing, weight-bearing heel lift (HL), and balance board. Though safe and effective in early rehabilitation, these exercises do not challenge PL performance of ankle eversion while maintaining postural balance and weight bearing. We believe that the optimal peroneal strength and control can be elicited during eversion of inverted ankle on a single-leg standing and such maneuver is important for functional performance of peroneal muscles. In this study, we tested an exercise that engages peroneal function in terms of eversion, balancing, and single-leg standing. We documented PL EMG and ankle movement. For a comparison, subjects also performed HL during one-legged stand, an exercise believed to evoke peak EMG activity in PL [8]. Each subject's maximal voluntary isometric contraction (MVIC) of PL was also recorded in order to ascertain the level of the maximal PL EMG activity that will be used to normalize EMG activity levels of HL and ankle eversion.

\section{Methods}

\subsection{Design}

We examined PL EMG levels, the dependant variable, in response to different ankle movements, the independent variables. The self-reported dominate leg was selected as the weight bearing leg. Subjects performed ankle eversion and heel lift during the one legged stand. To compare the activities of the PL muscle during ankle eversion and HL, PL EMG and ankle movements were monitored with a surface EMG electrode mounted over the PL muscle and an accelerometer affixed to the dorsum of the foot.

The eversion exercises were carried out by first placing the ankle joint in an inverted position. With the lateral edge of the foot rested on a force plate, the medial one third of the foot was elevated with a firm mat placed next to the force plate (Figure 1). For the eversion with low mat (EV1), a $8.5 \mathrm{~mm}$ thickness mat was used and created a 20 degree ankle inversion on average; a $17 \mathrm{~mm}$ thickness mat was deployed for the second eversion (EV2) resulting in an average 25 degree ankle inversion. The start and the end of each eversion were recorded when the lateral foot lifted and touched on the force plate respectively. The same weight bearing leg also performed HL. With the heel on the force plate and forefoot on the floor (Figure 2), the start and end of the movements were registered as the heel lifted off and touched down on the force plate. The ranges of ankle eversion or plantar flexion were recorded with an accelerometer that was pre-calibrated. Subjects were intrusted to move their ankle according to the verbal cadence of the researcher and to exert maximal effort to reach the limits of their ankle motion.

\subsection{Participants}

The experimental procedure and subject participation were approved by the institutional research ethics board. The twenty subjects, consisting of 7 male and 13 female volunteers, were recruited from the student population 


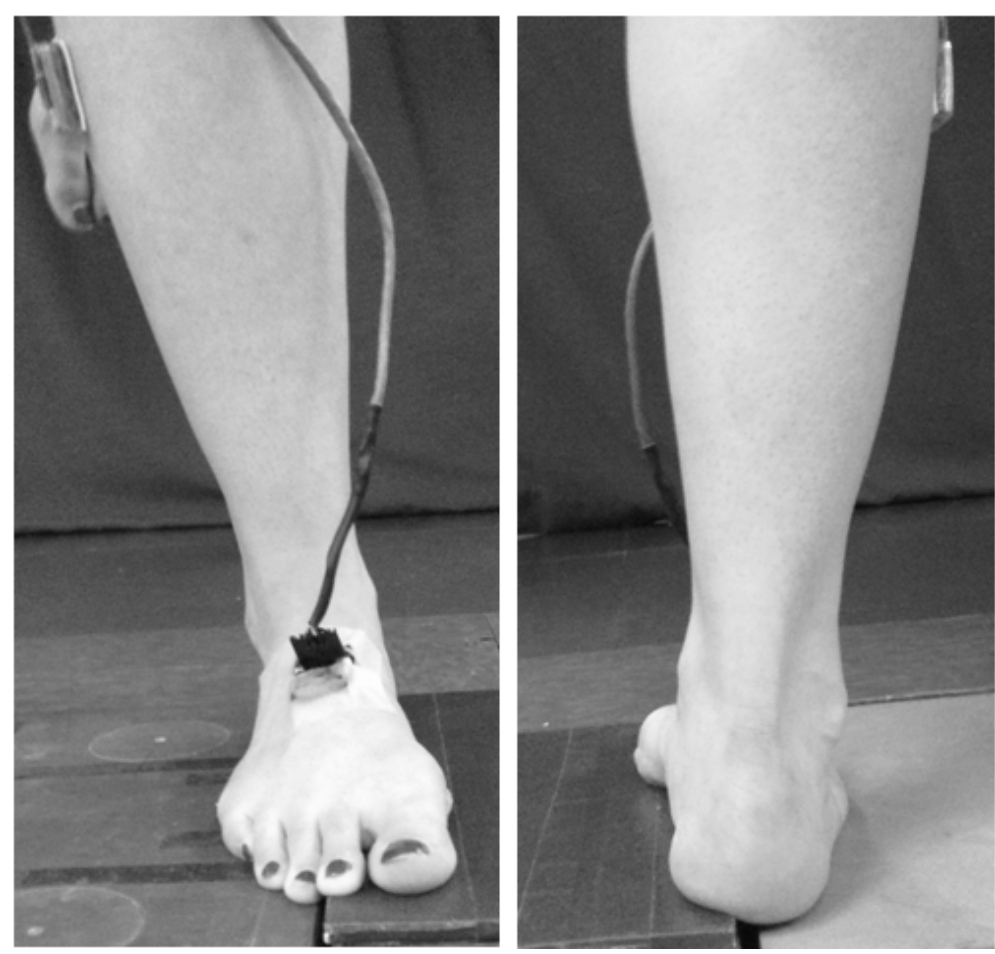

Figure 1. Eversions of the inverted ankle. To place the ankle into an inverted position, the medial one third of the foot was elevated with a firm matt right next to the force plate and the lateral edge rested on the force place. Two matt thicknesses were used, $8.5 \mathrm{~mm}$ thickness (EV1) and $17 \mathrm{~mm}$ (EV2), and created 20 degree and 25 degree inversions on average respectively. Peroneus longus EMG and ankle motion were monitored with a surface electrode, accelerometer, and a force place platform.

in school of human kinetics. All participants signed consent forms. The average age of the subjects was $20.5 \pm$ 1.5 , with a range of 19 to 24 . All subjects were free of ankle injury for at least 2 years, received no previous ankle specific training, and had no self-reported ankle instability episodes such as sudden giving way.

\subsection{Procedures}

PL muscle activity was recorded with a pre-amplified differential surface EMG electrode and electromyographic amplifier (Model 544, Therapeutics Unlimited Inc., Iowa City, IA). The surface electrode was placed on the skin at the junction of the proximal and middle third of the fibular over the palpable compartment [18]. Raw EMG voltages were band-pass filtered between $20 \mathrm{~Hz}-400 \mathrm{~Hz}$. These filtered voltages were then rectified and low-pass filtered at $6 \mathrm{~Hz}$ to create a linear envelope. MVIC of PL muscle was recorded first. MVIC was performed against manual resistance while the subject was in a sitting position and attempting ankle eversion and plantar flexion. For EMG recorded during ankle movements, the linear envelopes were normalized to the peak activity level of MVIC trials and expressed as percentage of MVIC. Average normalized EMG levels were determined for HL, EV1 and EV2. The range of ankle movement was monitored with an EVAL-ADXL327Z tri-axial accelerometer [Analog Devices, Nashua, $\mathrm{NH}$ ] that was affixed to the dorsum foot. Raw accelerometer voltages were converted to angles using a custom calibration performed prior to testing. An initial angular bias trial was recorded for each subject so that differences in the alignment of the accelerometer between subjects could be removed. To determine when the foot lifted off and re-established the contact on the force plate, vertical ground reaction forces were determined with an AMTI BP400600 force plate and a MSA-6 MiniAmp (Advance Mechanical Technology Incorporated, Watertown, MA). Forces less than $3 \mathrm{~N}$ were used to indicate when the subject's foot had lifted off the ground. This threshold was found to be optimal for the purposes of this study. 


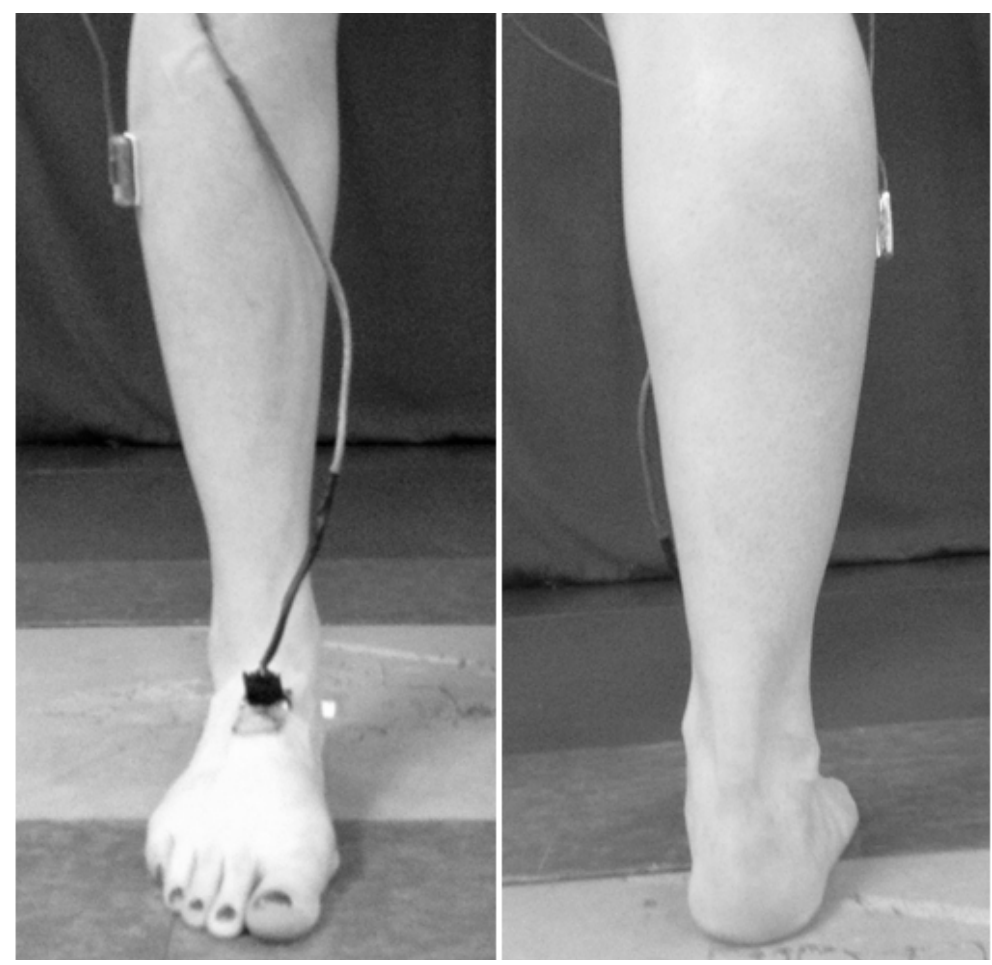

Figure 2. Heel lift. The heel lift of the weight bearing leg was carried with the heel on the force place and rest of the foot on the ground. Peroneus longus EMG and ankle motion were monitored with a surface electrode, accelerometer, and a force place platform.

For each type of the ankle movements as well as MVIC of PL muscle, subjects performed 3 trials. Each trial consisted of 3 consecutive eversions or heel lifts. Sample tracings for a typical trial were provided showing synchronous recordings of EMG and ankle motion data (Figure 3). The order of ankle movement type performed was randomized with a digital randomizer. While on one legged stand, subjects were only allowed to assist balancing with their fingertips, which could lightly touch a near by free standing frame if necessarily.

\subsection{Statistical Analysis}

A one way analysis of variance (ANOVA) with repeated measures was performed to assess the significance of the differences among the ankle movements. Post hoc paired $T$ tests were then conducted to evaluate the differences in pairs of the ankle movements. The alpha value for ANOVA analysis and the T tests were set at 0.01 and 0.05 respectively.

\section{Results}

The ranges of ankle motion were summarised in Table 1 commenced at two inverted ankle positions, the ankle movements ranged in 29 degrees of eversion or 31 degrees of eversion for EV1 and EV2 respectively. HL exercise produced 25 degrees of ankle planter flexion on average.

Expressed as the percentage of MVIC (Figure 4), the EMG linear envelope averages during the three ankle movements of the weight bearing leg were $64 \% \pm 7.25 \%$ (HL), $73 \% \pm 7.45 \%$ (EV1), and 74\% $\pm 7.07 \%$ (EV2). ANOVA revealed a significant difference among the EMG averages of the three ankle exercise, $\mathrm{F}[2.38]=6.471$, $\mathrm{P}<0.01$. Post hoc paired tests showed the averaged EMG linear envelopes of EV1 and EV2 were significantly higher than that of HL exercise and P values were $<0.014$ and 0.0016 for EV1 and EV2 respectively.

\section{Discussion}

In previous experiments, under non-weight bearing condition, the combined range of motion for active ankle 

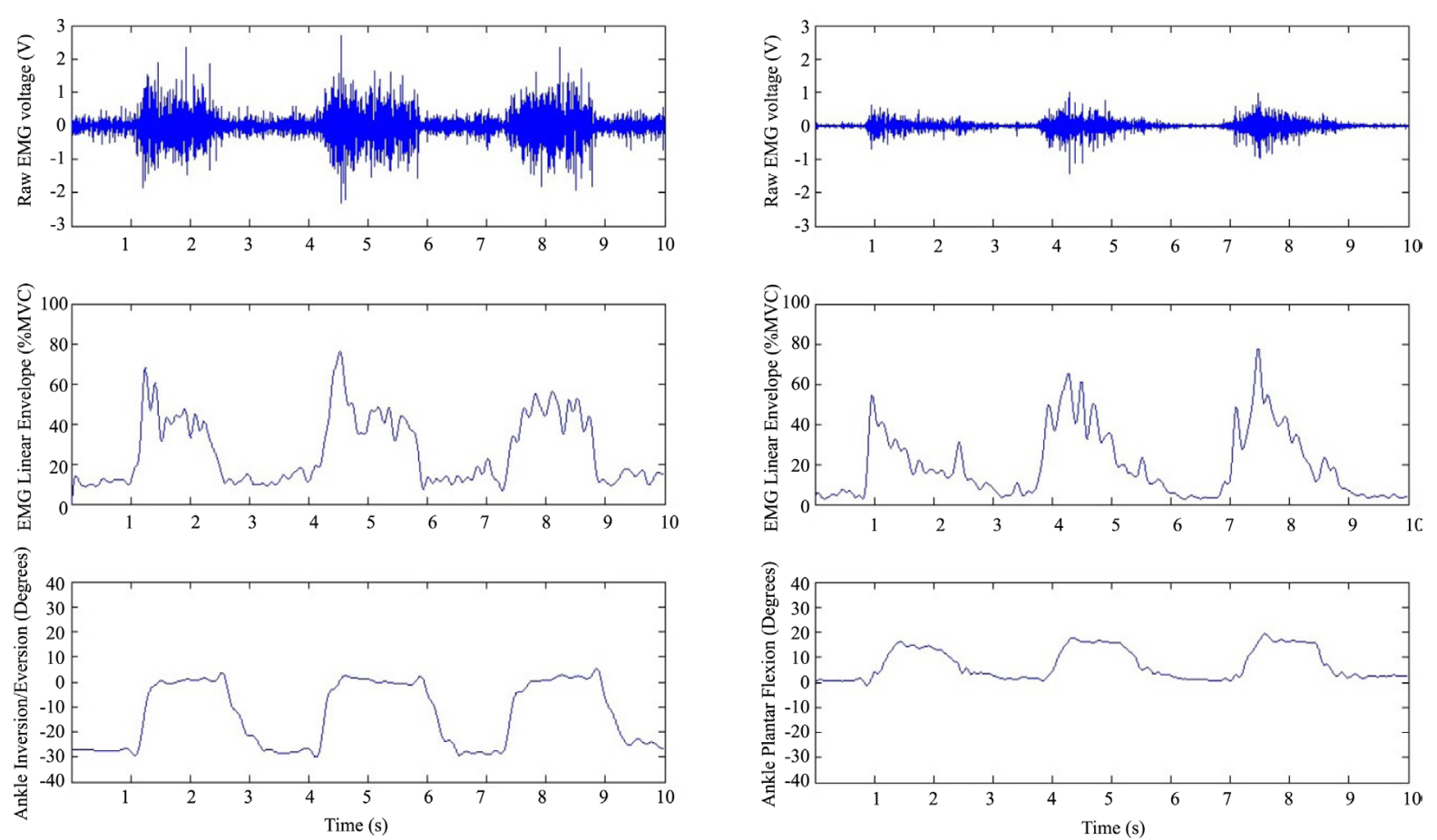

(a)

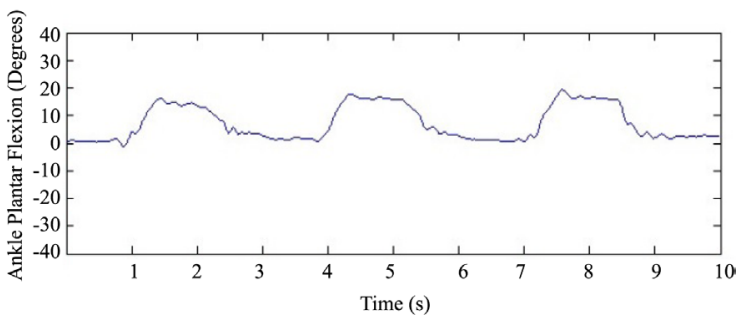

(b)

Figure 3. Sample tracings of peroneus longus EMG and ankle movements. Recordings made during ankle eversion (a) and heel lift (b). From top to bottom are raw EMG, linear envelope of EMG, and ankle movement in degrees.

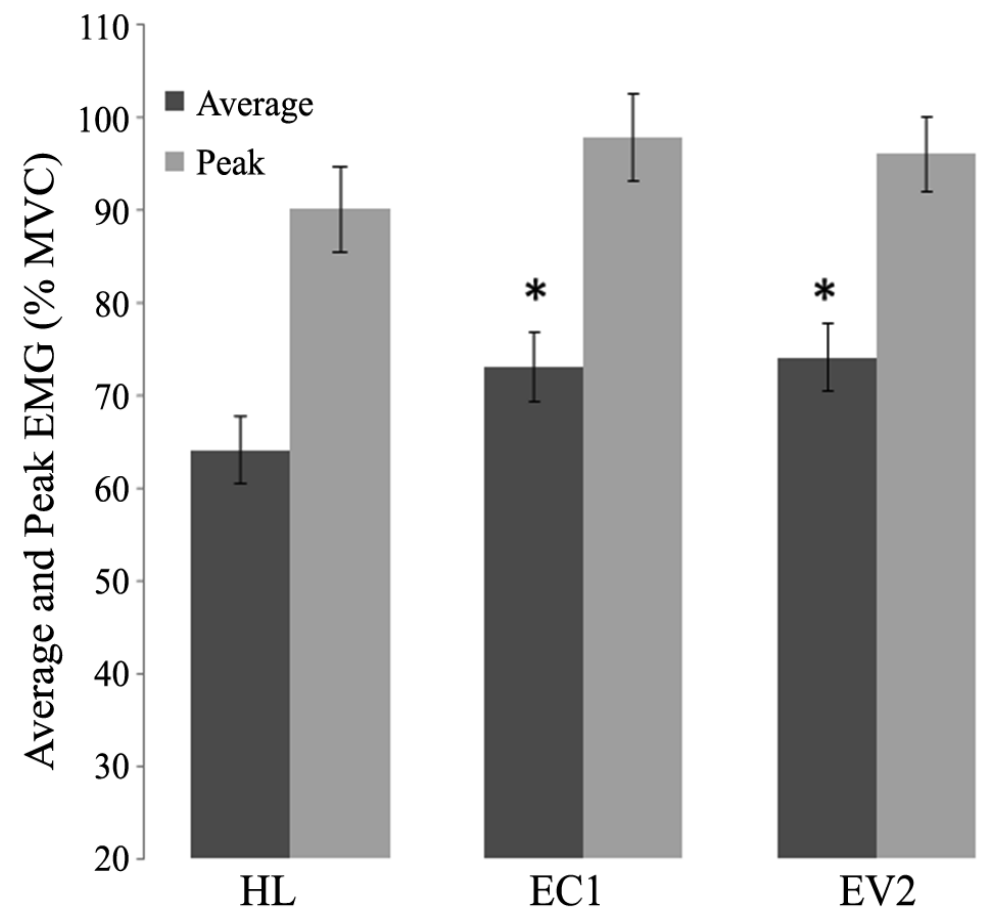

Figure 4. Average and Peak EMG Levels during ankle movement: peroneus longus activation is expressed as percentage of MVC. HL = heel lift, average $\mathrm{EMG}$ is $64 \%$; $\mathrm{EV} 1$ = eversion 1, average $\mathrm{EMG}$ is $73 \%$; $\mathrm{EV} 2$ = eversion 2, average EMG is $74 \%$. "Significant difference compared with HL, $\mathrm{P}<0.05$. Error bar = standard error of the mean. 
Table 1. Ankle range of motion.

\begin{tabular}{ccc}
\hline Ankle movement & Average ROM & Error \\
\hline HL & 25 & 1.6 \\
EV1 & 29 & 2.2 \\
EV2 & 31 & 2.4 \\
\hline
\end{tabular}

$\mathrm{HL}=$ heel lift; ROM = range of motion; Error = standard error of the mean. $\mathrm{N}=20$.

inversion and eversion was reported to be 42 degrees measured in sitting position with goniometer [19]. The ankle motion recorded in our study was smaller. Maintaining balance on a single leg stand put a restriction on extreme ankle inversion that may account for the smaller total ankle range of motion. However, total eversion ranges accomplished by subjects in this study are sufficiently large to produce meaningful ankle displacement and isotonic muscle contraction. The range of motion for heel lift recorded in this study also falls within the range of values reported by Lunsford and Perry [20].

Although HL of the weight bearing leg has been shown to elicit strong PL muscle activation in EMG and to be biomechanically relevant to forefoot stability during gait cycle, no comparison has been made to PL activation patterns of ankle eversion and HL in the weight bearing leg. We found EMG levels were significantly greater during ankle eversion. The strong peroneal activation recorded in this study was expected because of the combined tasks of balancing and eversion. Peroneal muscles are pivotal for ankle eversion and postural balance [2] [21], both of these functions are challenged while performing ankle eversion standing on one leg. HL exercise provoked less peroneal activity, likely due to the synergistic action of triceps surae group that partly mitigated the reliance on PL [22]. A similar comparison between vertical jump and side-cut during dynamic wholly body exercises also demonstrated side-cuts produced significantly greater surface EMG of PL than maximum vertical jumps [23].

PL is a primary stabilizer of the forefoot during gait cycle [9]. The peak activation of PL occurs during the push off phase when the increased rigidity of the forefoot is required [8]. These observations provided the impetus for the emphasis on HL exercise that strengthens peroneal muscles. Our results illustrated the need for more demanding eversion exercise of the ankle. In particular, in order to elicit peak peroneal activation, ankle exercise may need to incorporate balancing, weight bearing, and primary action(s) of the PL. Bellew et al. has shown a greater peroneal activation when a lateral pull was incorporated in HL exercise [7].

The benefit of the ankle eversion exercise described in this study may go beyond the strong peroneal activation. Activation and control of a muscle are not an isolated motor command but a part of a motor module or functional synergies of muscle groups over multiple joints recruited in a particular context of performance requirement [17] [24]. With or without ankle injury, muscular dysfunction may be more overt in the biomechanically relevant natural performance than strength deficiency measured in strength test. Consistent with this view were the findings that peroneal activity was significantly reduced during gait cycle while the concentric peroneal strength measured by instrument showed little or no weakness compared with the controls [3] [10]. Bloem et al. [25] showed that hip and trunk proprioceptive inputs may have contributed significantly to the initiation of lower leg balance correcting responses after a sudden perturbation of a platform on which subjects stood. These observations pointed to the importance of dynamic ankle control of evertor strength in balance and ankle injury. They also provided the support for the weight bearing ankle eversion in addition to non-weight bearing ankle eversion exercise and HL. Anankle conditioning exercise may achieve greater results if it engages the entire functional motor synergy in a specific task. Conversely, muscle synergy may be negatively affected after an event of injury as observed by Bullock-Saxton et al. [26]. They detected bilateral deficits of gluteus medius recruitment in subjects with a history of severe unilateral ankle sprain.

\section{Conclusion}

Exercises targeting PL have been fundamental in ankle conditioning and rehabilitation. Resistive ankle eversions and HL often constituted the core of ankle specific training before more dynamic movements of the entire lower extremities such as jumping and running. Our study provided quantified data and rationale in support of the weight bearing ankle eversion that can be incorporated in an ankle specific training. This exercise should be 
safe and practical to perform in view of the fact that only a small lift (8.5 mm thickness) under the medial foot was capable to elicit peak PL activation.

\section{References}

[1] Heckman, D.S., Reddy, S., Pedowitz, D., Wapner, K.L. and Parekh, S.G. (2008) Operative Treatment for Peroneal Tendon Disorders. Journal of Bone \& Joint Surgery, 90, 404-418. http://dx.doi.org/10.2106/JBJS.G.00965

[2] Ashton-Miller, J.A., Ottaviani, R.A., Hutchinson, C. and Wojtys, E.M. (1996) What Best Protects the Inverted Weightbearing Ankle Against Further Inversion? Evertor Muscle Strength Compares Favorably with Shoe Height, Athletic Tape, and Three Orthoses. American Journal of Sports Medicine, 24, 800-809. http://dx.doi.org/10.1177/036354659602400616

[3] Arnold, B.L., Linens, S.W., Motte, S.J. and Ross, S.E. (2009) Concentric Evertor Strength Differences and Functional Ankle Instability: A Meta-Analysis. Journal of Athletic Training, 44, 653-662. http://dx.doi.org/10.4085/1062-6050-44.6.653

[4] Vaes, P., Gheluwe, B.V. and Duquet, W. (2001) Control of Acceleration during Sudden Ankle Supination in People with Unstable Ankles. Journal of Orthopaedic \& Sports Physical Therapy, 31, 741-752. http://dx.doi.org/10.2519/jospt.2001.31.12.741

[5] Hopkins, T., Brown, T.N., Christensen, L. and Palmieri-Smith, R.M. (2009) Deficits in Peroneal Latency and Electromechanical Delay in Patients with Functional Ankle Instability. Journal of Orthopaedic Research, 27, 1541-1546. http://dx.doi.org/10.1002/jor.20934

[6] Payne, K.A., Berg, K. and Latin, R.W. (1997) Ankle Injuries and Ankle Strength, Fexibility, and Proprioception in College Basketball Players. Journal of Athletic Training, 32, 221-225.

[7] Gatev, P., Thomas, S., Kepple, T. and Hallett, M. (1999) Feedforward Ankle Strategy of Balance during Quiet Stance in Adults. Journal of Physiology, 514, 915-928. http://dx.doi.org/10.1111/j.1469-7793.1999.915ad.x

[8] Bellew, J.W., Frilot, C.F., Busch, S.C., Lamothe, T.V. and Ozane, C.J. (2010) Facilitating Activation of the Peroneus Longus: Electromyographic Analysis of Exercises Consistent with Biomechanical Function. Journal of Strength and Conditioning Research, 24, 442-446. http://dx.doi.org/10.1519/JSC.0b013e3181c088bc

[9] Louwerens, J.W.K., van Linge, B., de Klerk, L.W.L., Mulder, P.G.H. and Snijders, C.J. (1995) Peroneus Longus and Tibialis Anterior Muscle Activity in the Stance Phase. Acta Orthopaedica Scandinavica, 66, 517-523. http://dx.doi.org/10.3109/17453679509002306

[10] Santilli, V., Frascarelli, M.A., Paoloni, M., Frascarelli, F., Camerota, F., Natale, L. and De Santis, F. (2005) Peroneus Longus Muscle Activation Pattern during Gait Cycle in Athletes Affected by Functional Ankle Instability; A Surface Electromyography Study. American Journal of Sports Medicine, 33, 1183-1187. http://dx.doi.org/10.1177/0363546504274147

[11] Freeman, M.A.R. (1965) Instability of the Foot after Injuries to the Lateral Ligament of the Ankle. The Journal of Bone and Joint Surgery (British Volume), 47, 669-677.

[12] Hertel, J., Buckley, W.E. and Denegar, C.R. (2001) Serial Testing of Postural Control after Acute Lateral Ankle Sprain. Journal of Athletic Training, 36, 363-368.

[13] Tropp, H. and Odenrick, P. (1988) Postural Control in Single-Limb Stance. Journal of Orthopaedic Research, 6, 833839. http://dx.doi.org/10.1002/jor.1100060607

[14] Rozzi, S.L., Lephart, S.M., Sterner, R. and Kuligowski, L. (1999) Balance Training for Persons with Functionally Unstable Ankles. Journal of Orthopaedic \& Sports Physical Therapy, 29, 478-486. http://dx.doi.org/10.2519/jospt.1999.29.8.478

[15] Willems, T.M., Witvrouw, E., Delbaere, K., Mahieu, N., De Bourdeaudhuij, I. and De Clercq, D. (2005) Intrinsic Risk Factors for Inversion Ankle Sprains in Male Subjects: A Prospective Study. American Journal of Sports Medicine, 33, 415-423. http://dx.doi.org/10.1177/0363546504268137

[16] McGuine, T.A., Greene, J.J., Best, T. and Leverson, G. (2000) Balance as a Predictor of Ankle Injuries in High School Basketball Players. Clinical Journal of Sport Medicine, 10, 239-244. http://dx.doi.org/10.1097/00042752-200010000-00003

[17] Wang, H.K., Chen, C.H., Shiang, T.Y., Jan, M.H. and Lin, K.H. (2006) Risk-Factor Analysis of High School Basketball-Player Ankle Injuries: A Prospective Controlled Cohort Study Evaluating Postural Sway, Ankle Strength, and Flexibility. Archives of Physical Medicine and Rehabilitation, 87, 821-825. http://dx.doi.org/10.1016/j.apmr.2006.02.024

[18] Lynch, S.A., Eklund, U., Gottlieb, D., Renstrom, P.A. and Beynnon, B. (1996) Electromyographic Latency Changes in the Ankle Musculature During Inversion Moments. The American Journal of Sports Medicine, 24, 362-369. 
http://dx.doi.org/10.1177/036354659602400319

[19] Menadue, C., Raymond, J., Kilbreath, S.L., Refshauge, K.M. and Adams, R. (2006) Reliability of Two Goniometric Methods of Measuring Active Inversion and Eversion Range of Motion at the Ankle. BMC Musculoskeletal Disorders, 7, 60 .

[20] Lunsford, B.R. and Perry, J. (1995) The Standing Heel-Rise Test for Ankle Plantar Flexion: Criterion for Normal. Physical Therapy, 75, 694-698.

[21] Runge, C.F., Shupert, C.L., Horak, F.B. and Zajac, F.E. (1999) Ankle and Hip Postural Strategies Defined by Joint Torques. Gait \& Posture, 10, 161-170. http://dx.doi.org/10.1016/S0966-6362(99)00032-6

[22] Cresswell, A.G., Löscher, W.N. and Thorstensson, A. (1995) Influence of Gastrocnemius Muscle Length on Triceps Surae Torque Development and Electromyographic Activity in Man. Experimental Brain Research, 105, 283-290. http://dx.doi.org/10.1007/BF00240964

[23] Ozaki, T., Mizuno, K. and Grabiner, M.D. (1999) Peroneus Longus Can Not Be Fully Activated During Ankle Complex Exercises by Uninjured Subjects. The Kobe Journal of Medical Sciences, 45, 119-126.

[24] Torres-Oviedo, G.T. and Ting, L.H. (2010) Subject-Specific Muscle Synergies in Human Balance Control Are Consistent across Different Biomechanical Contexts. Journal of Neurophysiology, 103, 3084-3098. http://dx.doi.org/10.1152/jn.00960.2009

[25] Bloem, B.R., Allum, J.H., Carpenter, M.G., Verschuuren, J.J. and Honegger, F. (2002) Triggering of Balance Corrections and Compensatory Strategies in a Patient with Total Leg Proprioceptive Loss. Experimental Brain Research, 142, 91-107. http://dx.doi.org/10.1007/s00221-001-0926-3

[26] Bullock-Saxton, J.E., Janda, V. and Bullock, M.I. (1994) The Influence of Ankle Sprain Injury on Muscle Activation during Hip Extension. International Journal of Sports Medicine, 15, 330-334.

http://dx.doi.org/10.1055/s-2007-1021069 